Ophthalmologe 2018 $\cdot 115: 363-369$ https://doi.org/10.1007/s00347-018-0689-x Online publiziert: 18. April 2018

(c) Der/die Autor(en) 2018.

CrossMark

\author{
A. G. M. Jünemann ${ }^{1} \cdot$ R. Rejdak ${ }^{2} \cdot$ B. Hohberger ${ }^{3}$ \\ ${ }^{1}$ Klinik für Augenheilkunde, Universitätsmedizin Rostock, Universität Rostock, Rostock, Deutschland \\ ${ }^{2}$ Department of General Ophthalmology, Medical University of Lublin, Lublin, Polen \\ ${ }^{3}$ Augenklinik am Universitätsklinikum Erlangen, Friedrich-Alexander-Universität Erlangen-Nürnberg \\ (FAU), Erlangen, Deutschland
}

\title{
Trabekuläre mikroinvasive Glaukomchirurgie
}

\section{Verfahren und klinische Ergebnisse}

Die Glaukomerkrankung stellt uns als zweithäufigste Ursache für eine Erblindung vor große Herausforderungen. Bis zum heutigen Tag ist die genaue Pathogenese der Erkrankung unklar. Man geht davon aus, dass es sich um eine multifaktorielle Pathogenese handelt, bei welcher der intraokulare Druck (IOD) einen Hauptrisikofaktor darstellt. Alle therapeutischen Regime, ob konservativ oder chirurgisch, zielen daraufab, den IOD auf ein individuell angepasstes Zieldruckniveau zu senken, um die Verschlechterung dieser neurodegenerativen Erkrankung $\mathrm{zu}$ verlangsamen - aufzuhalten vermag man sie bis auf den heutigen Tag leider nicht [19]. Dies gilt sowohl für die Glaukomerkrankung mit erhöhtem Augeninnendruck als auch für Glaukome mit einem Augeninnendruck im sog. Normalbereich (Normaldruckglaukome). Jede Reduktion des IODs um einen einzelnen Punkt der mm Hg-Quecksilbersäule reduziert die Wahrscheinlichkeit um $12-13 \%$, dass der Patient eine Progression der glaukomatösen Optikusatrophie aufweist [23].

Konservative Therapien schließen teils komplexe Regime aus Mehrfachmedikationen mit ein, die neben einem hohen Allergiepotenzial auch die Schwierigkeit der Realisierung im Alltag für den Patienten mit sich bringen. Über medikamentöse Interaktionen, zum Teil sicherlich auch über die konservierungsmittelbedingten Benzalkoniumchlorid(BAC)-Years (Anzahl der BAC-Tropfen/Tag $\times$ Jahre), wird eine allergisch bedingte Blepharokonjunktivitis induziert, sodass anschließende chirurgische Verfahren mit einer „angegriffenen“ Konjunktiva zu kämpfen haben. Vernarbungsreaktionen sind nur allzu oft die Folge und führen zu erneuten Operationen. Ist über konservative Behandlungswege eine adäquate Senkung des IODs nicht mehr zu erreichen bzw. überwiegen die medikamentösen Nebenwirkungen der Lokaltherapie, so kommt die Chirurgie zum Tragen. In diesem Bereich hat in den letzten Jahren die mikroinvasive Glaukomchirurgie (MIGS) zunehmend Bedeutung gewonnen. Mit diesen chirurgischen Techniken kann neben einer signifikanten IOD-Senkung auch eine Reduktion der lokalen bzw. systemischen antiglaukomatösen Therapie erzielt werden. Zudem wird die intra- bzw. postoperative Verweildauer der Patienten verringert, sodass die teils aufwendigen Nachkontrollen bzw. langwierigen Therapieregime deutlich reduziert bzw. vereinfacht werden können.

\section{》) Die mikroinvasive Glaukomchirurgie hat zunehmend an Bedeutung gewonnen}

Eine einheitliche Definition des Terminus „MIGS“ findet man in der Literatur bislang noch nicht. Im Jahr 2014 wurde „MIGS“ von einem Arbeitskreis der American Glaucoma Society and the US Food and Drug Administration (FDA) definiert als Überbegriff für chirurgische Verfahren, die den IOD ab interno oder ab externo drainieren - mit nur geringer bis vollständig fehlender Skleradissektion [6]. Dabei soll eine IOD-Senkung von mindestens $20 \%$ bzw. $3 \mathrm{~mm} \mathrm{Hg}$ erzielt werden [6]. Eine etwas weiterführende Definition findet man in der Publikation von Saheb und Ahmed [29]: Unter dem Begriff „MIGS“ werden chirurgische Verfahren gruppiert, die ab interno über eine korneale Inzision signifikant den IOD zu senken vermögen, ohne dass die Konjunktiva hierfür eröffnet werden muss. Neben einem nur geringen intraoperativen Trauma weisen die MIGS-Verfahren ein hohes Sicherheitsprofil auf und ermöglichen eine schnelle Rekonvaleszenz für den Patienten. Aufgrund ihres additiven, deutlich reduzierten postoperativen Komplikationsprofils (z.B. Hypotonie) sind die MIGS-Verfahren gut geeignet, in Kombination mit einer Phakoemulsifikation und Implantation einer Intraokularlinse (PE/IOL) operiert zu werden.

Allen Verfahren ist gemeinsam, dass sie das Kammerwasser aus der Vorderkammer über verschiedene Drainagewege ableiten - transtrabekulär [28, $37,38]$, in den suprachoroidalen [18] oder subkonjunktivalen Raum ([20, 24]; - Tab. 1). Dieser Reviewbeitrag möchte einen Überblick über die aktuell vorhandenen MIGS-Implantate für die trabekuläre Drainage geben und diese im Rahmen der aktuell vorhandenen Literatur diskutieren. 
Tab. 1 Überblick über Implantate der MIGS(mikroinvasive Glaukomchirurgie)-Verfahren

\begin{tabular}{|c|c|c|c|c|c|c|}
\hline \multirow[b]{2}{*}{ Implantat } & \multicolumn{3}{|l|}{ Trabekuläre Drainage } & \multirow{2}{*}{$\begin{array}{l}\text { Subkonjunktivale } \\
\text { Drainage } \\
\text { Xen45 Gel Stent }\end{array}$} & \multicolumn{2}{|c|}{ Suprachoroidale Drainage } \\
\hline & iStent & iStent inject & $\begin{array}{l}\text { Hydrus Mi- } \\
\text { krostent }\end{array}$ & & Cypass & iStent supra \\
\hline Hersteller & $\begin{array}{l}\text { Glaukos Inc. Laguna, } \\
\text { Hills, CA, USA }\end{array}$ & $\begin{array}{l}\text { Glaukos Inc. Laguna, } \\
\text { Hills, CA, USA }\end{array}$ & $\begin{array}{l}\text { Ivantis, Inc., } \\
\text { Irvine CA, USA }\end{array}$ & $\begin{array}{l}\text { Pharm-Allergan } \\
\text { GmbH (Allergan, } \\
\text { Irvine, CA, USA) }\end{array}$ & $\begin{array}{l}\text { Alcon Inc. Fort } \\
\text { Worth, TX, USA }\end{array}$ & $\begin{array}{l}\text { Glaukos Inc. Laguna, } \\
\text { Hills, CA, USA }\end{array}$ \\
\hline Material & $\begin{array}{l}\text { Heparinbeschichtetes } \\
\text { Titan }\end{array}$ & $\begin{array}{l}\text { Heparinbeschichtetes } \\
\text { Titan }\end{array}$ & $\begin{array}{l}\text { Nickel-Titan- } \\
\text { Legierung }\end{array}$ & $\begin{array}{l}\text { Tierische Gelatine } \\
\text { (Schwein) }\end{array}$ & Polyamid & $\begin{array}{l}\text { Polyethersulfon in } \\
\text { Kombination mit } \\
\text { Titan }\end{array}$ \\
\hline Länge (mm) & 1 & 0,32 & 8 & 6 & 6,35 & 4 \\
\hline $\begin{array}{l}\text { Innendurch- } \\
\text { messer ( } \mu \mathrm{m})\end{array}$ & 120 & 230 & $185-292$ & 45 & 300 & 165 \\
\hline
\end{tabular}

\section{Trabekuläre Drainage}

Die trabekulären Implantate drainieren in den Hauptabflussweg des Kammerwassers, den Schlemm-Kanal, indem sie das Trabekelmaschenwerk umgehen. Nach dem aktuellen Stand der Forschung liegt der Hauptabflusswiderstand bei Patienten mit primärem Offenwinkelglaukom in dem kontraktilen Trabekelmaschenwerk [33]. Aktuell gibt es 3 Implantate mit CE-Zertifizierung zur Umgehung des trabekulären Widerstandes: iStent ${ }^{\circledR}$ (Glaukos Inc. Laguna, Hills, CA, USA), iStent inject ${ }^{\oplus}$ (Glaukos Inc. Laguna, Hills, CA, USA) und Hydrus (Ivantis, Inc., Irvine, CA, USA), von denen die beiden ersten kommerziell erhältlich sind. Mit diesen Stents kann eine Reduktion des IODs bis auf Werte im Bereich des episkleralen Venendruckes erzielt werden [7, 32].

\section{iStent $^{\circledR}$}

Der iStent $^{\circledR}$ (Glaukos Corporation, 229 Avenida Fabricante, San Clemente, CA 92672 USA, www.glaukos.com) der ersten Generation ist ein $1 \mathrm{~mm}$ langes heparinbeschichtetes Titanimplantat mit einer L-förmigen Konfiguration und einem Innendurchmesser von $120 \mu \mathrm{m}$ (- Abb. 1). Es war das erste Implantat, das auf dem US-Markt zugelassen wurde. Dieses Implantat wird ab interno über einen kornealen Zugang unter gonioskopischer Kontrolle in den Schlemm-Kanal implantiert. Postoperativ schließen sich eine antiinflammatorische sowie antibiotische Lokaltherapie für 4 Wochen an. Seit 2012 sind Kombinationseingriffe mit einer PE/IOL von der FDA zugelassen, in Europa auch als Stand-aloneChirurgie.

Daten bezüglich der Effektivität des iStent sind in großer Menge vorhanden - ob als Stand-alone-Technik von einem iStent, der Implantation mehrere iStents sowie als Kombinationseingriffe mit einer PE/IOL.

Die erste Studie aus dem Jahr 2009 erbrachte eine IOD-Senkung um 4,6 $\mathrm{mm} \mathrm{Hg}$ (21\%) bei einer Verminderung der Antiglaukomatosa um 1,2\% für einen kombinierten Eingriff aus iStent und PE/IOL nach 1 Jahr Nachbeobachtung [31]. In den Studien der darauffolgenden Jahre wurde für Kombinationseingriffe aus einem iStent und PE/IOL eine IOD-Reduktion um $1,3 \mathrm{~mm} \mathrm{Hg}$ (12 Augen) [10], 1,5 $\mathrm{mm} \mathrm{Hg}$ (111 Augen) [30], $1,9 \mathrm{~mm} \mathrm{Hg}$ (10 Augen) [13], 3,2 mm Hg (19 Augen) [2], 3,8 $\mathrm{mm} \mathrm{Hg}$ (10 Augen) [35], 4,8 $\mathrm{mm} \mathrm{Hg}$ (44 Augen) [27] und $9,2 \mathrm{~mm} \mathrm{Hg}$ (62 Augen) [26] beschrieben. Eine additive Verringerung der Antiglaukomatosa fand sich mit Werten zwischen $0,48(36,4 \%)$ [2] und 2 (100\%) [10]. Zwei Studien betrachteten den IOD-senkenden Effekt einer Standalone-iStent-Implantation [5, 36]. Zeigte sich in der Studie von Buchacra et al. [5] eine IOD-Senkung um $9,5 \mathrm{~mm} \mathrm{Hg}$ $(35,8 \%)$ bei einer gleichzeitigen Reduktion der Antiglaukomatosa um 1,8 (62,1\%) nach 1 Jahr Nachbeobachtung, konnten Vold et al. [36] eine IOD-Reduktion um $4,2 \mathrm{~mm} \mathrm{Hg}(21,2 \%)$ bei einer Reduktion der Antiglaukomatosa um 1,5 (89,5\%) nach 18 Monaten erreichen.

Implantiert man mehrere iStents, scheint sich ein additiver Effekt der
IOD-Senkung abzuzeichnen. Eine Standalone-Implantation von 2 iStents erzielte eine IOD-Reduktion von $5,1 \mathrm{~mm} \mathrm{Hg}$ [1, 25] bis $10,9 \mathrm{~mm} \mathrm{Hg}$ [36] bei einer Verminderung der Antiglaukomatosa um 0,95 bis $1,6[9,21]$. Führte man diesen Eingriff simultan mit einer PE/IOL durch, zeigten sich IOD-Werte, die um bis $\mathrm{zu} 6,6 \mathrm{~mm} \mathrm{Hg}$ verringert waren mit einer Reduktion der Antiglaukomatosa um $100 \%$ [15]. Nach einer Implantation von 3 iStents wurde eine IOD-Reduktion um $8,3 \%$ mit einer Verminderung der Antiglaukomatosa um 94,7\% erzielt [21]. Vergleicht man die Effektivität von 2 mit 3 implantierten iStents, konnten Belovay et al. [4] zeigen, dass 3 iStents den IOD um $20 \%$ sowie die Antiglaukomatosa um $85 \%$ senken konnten, wohingegen nach einer Implantation von 2 iStents der IOD-senkende Effekt gleich (20\%), die Verminderung der Antiglaukomatosa jedoch geringer war $(64 \%)$.

\section{》) Implantiert man mehrere iStents, scheint sich ein additiver Effekt der IOD-Senkung abzuzeichnen}

iStents werden als Implantate mit einem hohen Sicherheitsprofil angesehen $[3,13$, 25]. Vorübergehende, meist selbstlimitierende Hyphämata sind die noch am meisten in der Literatur beschriebenen Komplikationen $[9,34,36]$. Sehr selten findet man postoperative IOD-Spitzen $[10,14$, 30] und Stentobstruktionen oder -verlagerungen $[8,31,36]$. Eine Studie beob- 
Hier steht eine Anzeige.

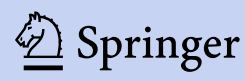


achtete das vermehrte Auftreten von Katarakten nach implantierten iStents [1].

\section{iStent inject ${ }^{\circledR}$}

Als Implantat der zweiten Generation wurde der iStent modifiziert, um eine einfachere Implantation von bis $\mathrm{zu}$ 2 Stents über einen Injektor zu ermöglichen. Mit einer Länge von $0,36 \mathrm{~mm}$ und einem Durchmesser von $230 \mu \mathrm{m}$ ist der iStent inject ${ }^{\circledR}$ (Glaukos Corporation, 229 Avenida Fabricante, San Clemente, CA 92672 USA, www.glaukos.com) zum aktuellen Zeitpunkt das kleinste zugelassene Implantat in der Medizin (• Abb. 2). Wie bei dem iStent werden unter gonioskopischer Kontrolle über eine Clearcornea-Inzision 1 bzw. 2 iStent inject in einem Abstand von $30-60^{\circ}$ in dem Kammerwinkel implantiert. Postoperativ werden antiinflammatorische sowie antibiotische Augentropfen appliziert.

Implantiert man einen iStent inject, findet man eine IOD-Reduktion von $7 \mathrm{~mm} \mathrm{Hg}$ [17] sowie $8,4 \mathrm{~mm} \mathrm{Hg}$ [22] bei einer Antiglaukomatosareduktion von $55,4-59,8 \%$ [17, 22]. Der IODsenkende Effekt von 2 iStent inject wird mit Werten von $6,4 \mathrm{~mm} \mathrm{Hg}$ (Antiglaukomatosareduktion: 87,3\%; [37]) bis $8,1 \mathrm{~mm} \mathrm{Hg}$ (Antiglaukomatosareduktion: $91,5 \%$; [12]) beschrieben. Bis zu $42 \%$ IOD-Reduktion, bezogen auf den präoperativen IOD, wurden nach Implantation von 2 iStent inject erzielt [25]. Eine Kombination aus 2 iStent inject und PE/IOL zeigte eine IOD-Senkung von $34 \%$ (Antiglaukomatosareduktion: $36 \%$; [17]).

Die postoperative Komplikationsrate von iStent inject und iStent sind vergleichbar. Beide Mikrostents zeichnen sich durch ein hohes Sicherheitsprofil aus, wobei es in seltenen Fällen zu einer Verlegung oder Verlagerung des Implantates kommen kann. Weiterhin ist positiv $\mathrm{zu}$ bewerten, dass für iStent und iStent inject in der Literatur bislang keine Berichte über Hypotonien oder Endothelzellverluste, die wiederum im weiteren Verlauf zu einer Hornhautepithel-Endothel-Dekompensation führen können, zu finden sind.

Ophthalmologe 2018 $\cdot 115: 363-369$ https://doi.org/10.1007/s00347-018-0689-x (c) Der/die Autor(en) 2018.

\section{A. G. M. Jünemann · R. Rejdak • B. Hohberger}

\section{Trabekuläre mikroinvasive Glaukomchirurgie. Verfahren und klinische Ergebnisse}

\section{Zusammenfassung}

Bislang verläuft der typische Weg eines

Glaukompatienten von der medikamentösen

Einfachtherapie, über Mehrfachtherapi-

en und Lasereingriffe zur filtrierenden

Glaukomchirurgie. Nicht nur die additiven

Nebenwirkungen der Mehrfachtherapie, auch

die mangelnde Adhärenz und Persistenz

der Patienten und das Komplikationsrisiko

der herkömmlichen Glaukomchirurgie

begründen die Einführung neuer operativer

Verfahren. Die Entwicklung der mikro-

invasiven Glaukomchirurgie (MIGS) lässt

hier auf einen neuen Behandlungsweg

hoffen. Mikroimplantate können helfen, eine
Mehrfachtherapie zu vermeiden und den filtrierenden Eingriff zu verzögern. In dieser Übersichtsarbeit werden die Implantate für die trabekuläre Drainage (iStent ${ }^{\circledR}$ [Glaukos Inc. Laguna, Hills, CA, USA], iStent inject ${ }^{\circledR}$ [Glaukos Inc. Laguna, Hills, CA, USA], Hydrus ${ }^{\mathrm{Tm}}$ Microstent [Ivantis Inc, Irvine, CA, USA]) im Detail vorgestellt, und die aktuelle Datenlage zur Effektivität und Sicherheit wird diskutiert.

Schlüsselwörter

Mikroimplantat · Trabekuläre Drainage · Sicherheit · Effektivität · Augeninnendruck

\section{Trabecular micro-invasive glaucoma surgery. Procedures and clinical results}

\begin{abstract}
The management of glaucoma therapy to reduce intraocular pressure commonly consists of a gradual approach with local monotherapy, combined therapy, laser surgery and finally filtration surgery. The local side effects of glaucoma medications and the lack of adherence and persistence to the medical therapy as well as the complication profile of the established glaucoma surgical techniques justify the introduction of new surgical procedures. Micro-invasive glaucoma surgery (MIGS) is a promising new surgical
\end{abstract}

approach. Microstents can reduce the medication burden and prolong the need for filtration surgery. This review article presents the different trabecular implants (iStent, iStent inject, Hydrus ${ }^{\mathrm{TM}}$ Microstent) in detail and discusses the effectiveness and safety of the procedures based on the currently available data.

Keywords

Microimplant - Trabecular drainage · Safety . Effectiveness · Intraocular pressure

\section{Hydrus $^{\mathrm{TM}}$ Microstent}

Der Hydrus ${ }^{\mathrm{TM}}$ Microstent (Ivantis, Inc., 38 Discovery, Suite 150, Irvine, CA 92618 , www.invantisinc.com) besteht aus einer Nickel-Titan-Legierung. Mit einer Länge von $8 \mathrm{~mm}$ sowie einem Innendurchmesser von 185-292 $\mu \mathrm{m}$ wird der Stent $a b$ interno über eine Clear-cornea-Inzision in den Schlemm-Kanal implantiert (• Abb. 3). Das Implantat steht derzeit nur für Studien zur Verfügung. Durch den Hydrus ${ }^{\mathrm{TM}}$ Microstent wird der nasale Anteil des Kammerwinkels um bis zu 3 Uhrzeiten aufgedehnt, sodass eine verbesserte Kammerwasserdrainage ermöglicht wird. Postoperativ werden antiinflammatorische sowie antibiotische $\mathrm{Au}$ - gentropfen appliziert. Auch mit diesem transtrabekulären Mikrostent sind Kombinationseingriffe mit einer PE/IOL möglich [28].

In einer randomisierten klinischen Studie (HYDRUS II) [28] konnte eine Reduktion des präoperativen IODs von $26,3 \pm 4,4 \mathrm{mmHg}$ auf Werte von $16,9 \pm 3,3 \mathrm{mmHg}$ postoperativ sowie eine Verminderung der Anzahl an Antiglaukomatosa von $2 \pm 1$ auf $0,5 \pm 1$ in der Kombinationsgruppe aus Hydrus ${ }^{\mathrm{TM}}$ Microstent und PE/IOL erzielt werden. Sie zeigten einen signifikant niedrigeren IOD als die Kontrollgruppe, bestehend aus Patienten, die nur eine PE/IOL erhalten hatten $(19,2 \pm 4,7 \mathrm{~mm} \mathrm{Hg})$. Der Vergleich zu der selektiven Lasertra- 


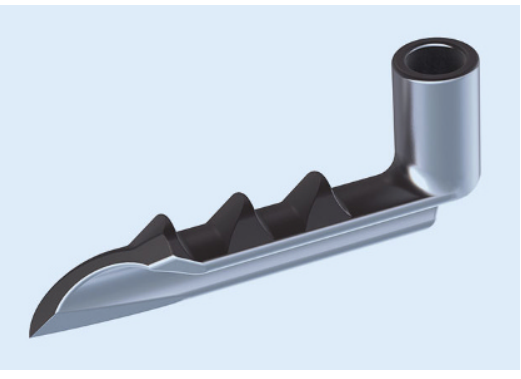

Abb. $1 \Delta$ iStent ${ }^{\circledR}-$ Implantat: 1 mm langes heparinbeschichtetes Titanimplantat mit einer L-förmigen Konfiguration und einem Innendurchmesser von $120 \mu \mathrm{m}$. (Mit freundl. Genehmigung von Glaukos Corporation, Inc. Laguna, Hills, CA, USA)

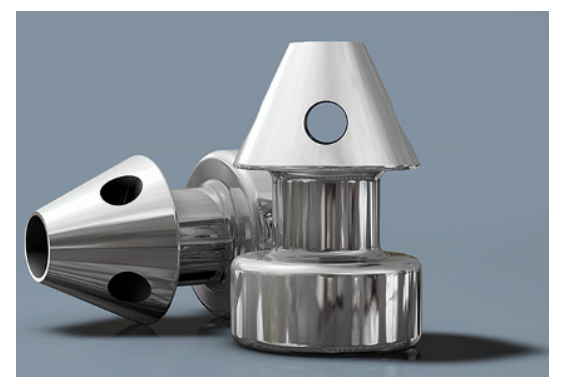

Abb. $2 \Delta$ iStentinject ${ }^{\circledR}-$ Implantat: $360 \times 230 \mu \mathrm{m}$ großes, heparinbeschichtetes Titanimplantat mit einem Innendurchmesser von $80 \mu \mathrm{m}$ und 4 Öffnungen mit einem Innendurchmesser von $50 \mu \mathrm{m}$. (Mit freundl. Genehmigung von Glaukos Corporation, Inc. Laguna, Hills, CA, USA)

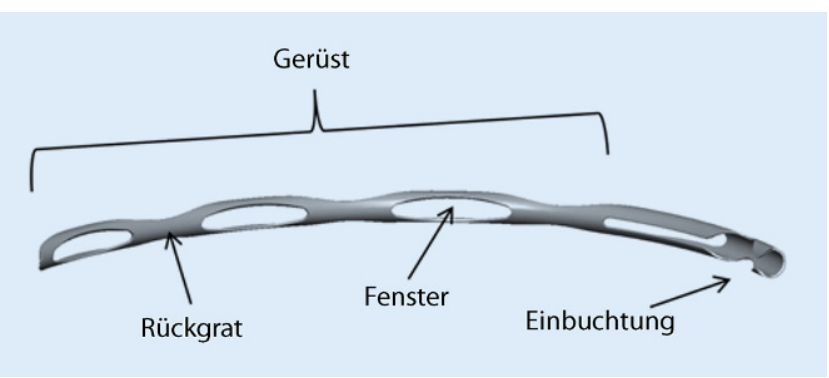

Abb. 3 ॥ Hydrus ${ }^{T M}$ Microstent: Implantat aus einer Nickel-Titan-Legierung mit einer Länge von 8 mm sowie einem Innendurchmesser von 185-292 $\mu \mathrm{m}$. (Aus [39]; mit freundl. Genehmigung von Wolters Kluwer Health Inc., Promotional and commercial use of the material in print, digital or mobile device format is prohibited without the permission from the publisher Wolters Kluwer. Please contact permissions@lww.com for further information.)

bekuloplastik (SLT) erbrachte einen ähnlich IOD-senkenden Effekt (Hydrus: $23,1 \pm 5,08 \mathrm{~mm} \mathrm{Hg}$ auf $16,5 \pm 2,6 \mathrm{~mm} \mathrm{Hg}$ vs. SLT: $23,2 \pm 2,15 \mathrm{~mm} \mathrm{Hg}$ auf $15,9 \pm$ $2,49 \mathrm{~mm} \mathrm{Hg}$ ) bei jedoch deutlich verringerter Lokaltherapie $(-1,4 \pm 0,97$ vs. $-0,5 \pm 1,05)$ [11].

Bislang sind keine schwerwiegenden Nebenwirkungen sowie Komplikationen nach Implantation eines Hydrus ${ }^{\mathrm{TM}}$ Microstent beschrieben worden. Vereinzelt finden sich IOD-Spitzen in der frühen postoperativen Phase [11, 16] sowie selbst-resorbierende Hyphämata [16] und fokale vordere Synechien, die zum Teil mittels Yttrium-AluminiumGranat(YAG)-Lasers gelöst wurden [16], zum Teil in einer anderen Studie keiner weiteren Intervention bedürftig waren [28].

\section{Indikation}

Im Wesentlichen bestimmen 3 Aspekte die Indikation: die Konfiguration des
Kammerwinkels, das Stadium der Glaukomerkrankung und somit der Zieldruck und die Medikamentenlast. Trabekuläre MIGS-Chirurgie mit den genannten Implantaten kann nur bei Offenwinkelglaukomen Anwendung finden. Voraussetzung für die Empfehlung zu dieser Chirurgie ist somit die Gonioskopie (• Abb.4) mit Nachweis eines offenen Kammerwinkels.

\section{》) Der Indikationsbereich liegt bei einem Zieldruck von $15 \mathrm{~mm} \mathrm{Hg}$}

Da die trabekuläre MIGS-Chirurgie nur den trabekulären Widerstand umgeht, das Kammerwasser nach Eintritt in den Schlemm-Kanal durch das augeneigene Abflusssystem fließt, kann der IOD nicht auf ein so niedriges Niveau sinken wie bei filtrierender Chirurgie, bei der alle seriell geschalteten Abflusswiderstände bis in den subtenonalen Raum eröffnet werden. Somit liegt der Indikationsbereich bei einem Zieldruck von $15 \mathrm{~mm} \mathrm{Hg}$, auch wenn individuell durchaus niedrigere IOD-Werte erzielt werden können.

Die Reduzierung der Anzahl der lokal applizierten Antiglaukomatosa stellt den dritten wesentlichen Aspekt zur Indikation dar. Dies ist insofern von großer Bedeutung, als eine langjährige Tropfenapplikation zu einer Alteration des $\mathrm{Ab}$ flusssystems mit erhöhtem Abflusswiderstand auch jenseits des Schlemm-Kanals führen kann. Neben der rein morphologischen Gonioskopie ist eine funktionelle Gonioskopie (• Abb. 5) mit Beurteilung des Kammerwasserrefluxes zur optimierten Indikationsstellung notwendig. Eine möglichst nichtinvasive präoperative Kanalographie stellt hier die diagnostische Herausforderung für die Zukunft dar.

\section{Perioperatives Management}

Eine präoperative Vorbereitung des Auges wie bei den filtrierenden Operationen (Trabekulektomie, XEN-Gelimplantat) ist bei den trabekulären Mikrostents bisher nicht empfohlen. Es gibt keine $\mathrm{Da}$ ten zu der Frage, ob das Absetzen der drucksenkenden Augentropfen und die Applikation von BAC-freien, steroidhaltigen Augentropfen (unter Druckregulierung mit systemischen Carboanhydrasehemmern) für eine oder mehrere Wochen präoperativ das Operationsergebnis positiv beeinflussen. Die Implantationen können unter der antikoagulativen Therapie des Patienten durchgeführt werden. Die postoperative Therapie besteht aus antibiotischen Augentropfen für eine Woche und lokalen Steroiden, die wöchentlich reduziert werden. Es sind BACfreie Augentropfen beim Glaukompatienten anzustreben.

\section{Vergleichsstudien}

Um die Effektivität und Sicherheit der trabekulären Implantate im breiten Spektrum der Glaukomchirurgie im Hinblick auf die Indikation sicher beurteilen $\mathrm{zu}$ können, sind vergleichende Studien zwischen den einzelnen MIGS-Verfahren bzw. mit der herkömmlichen Glaukomchirurgie notwendig. In einer intraindividuellen Vergleichsstudie wurde die 


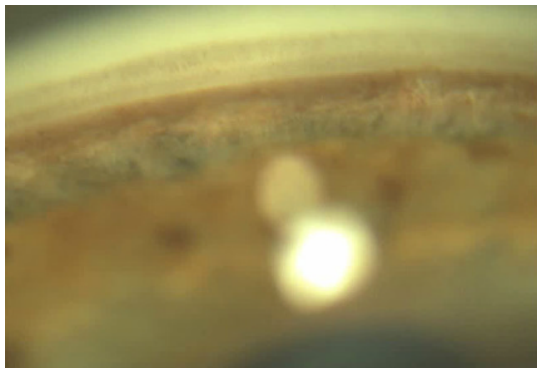

Abb. 4 \& Offener Kammerwinkel: Identifizierung von Skleralsporn, Schwalbe-Linie als Leitlinien sowie Trabekelmaschenwerk und Iriswurzel (Jünemann, mit freundl. Genehmigung)

IOD-Senkung des iStent inject und des Trabektoms in Kombination mit mikroinzisioneller Phakoemulsifikation verglichen [17]. Die retrospektive Auswertung betrachtete 54 Augen von 27 Patienten mit mildem bis moderatem primärem Offenwinkelglaukom (POWG) und sekundärem Offenwinkelglaukom (OWG) bei Pseudoexfoliationssyndrom (PEXG). Die IOD-Reduktion nach 12 Monaten betrug für den iStent inject $34 \%$, für das Trabektom $30 \%$ (ohne signifikanten Unterschied). Auch die Reduzierung der lokalen Antiglaukomatosa wies keinen signifikanten Unterschied auf, auch wenn sie für den iStent im Vergleich zum Trabektom etwas größer (37,3 vs. $30,8 \%)$ war [17].

Eine zweite retrospektive, nicht randomisierte vergleichende Fallserie von 45 Patienten mit Offenwinkelglaukomen (POWG, PEXG, sekundäres OWG bei primärem Melanindispersionssyndrom) untersuchte den IOD-senkenden Effekt und die Sicherheit von Hydrus $(n=21)$ und Kanaloplastik $(n=24) 2$ Jahre postoperativ [16]. Die prozentuale IOD-Senkung war für Hydrus und Kanaloplastik vergleichbar (37,5\% vs. $38,4 \%)$. Hyphämata als einzige postoperative Komplikation traten bei der Kanaloplastik etwas häufiger auf $(29,2 \%)$ als beim Hydrus (19\%), erforderten jedoch keine Intervention. Bei beiden Verfahren waren 2 Jahre postoperativ Visus und funktioneller Glaukomschaden (gemessen anhand des "mean defect" in der Perimetrie) vergleichbar und unverändert. Die Autoren machten jedoch keine Angaben $\mathrm{zu}$ Visusveränderungen in der frühen postoperativen Phase infolge eines Astigmatismus bei der Kanaloplastik oder zu

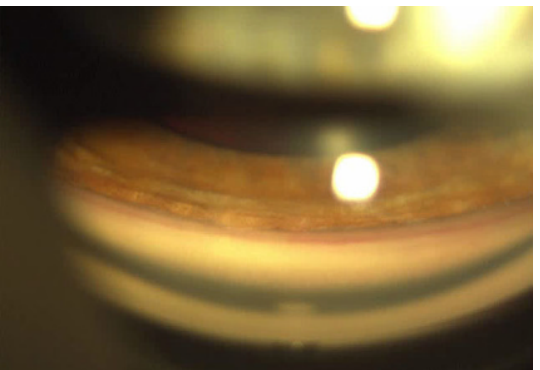

Abb. 5 Funktionelle Gonioskopie: Rotfärbung des Schlemm-Kanals als Hinweis für Blutreflux bei hypotonem Auge (Jünemann, mit freundl. Genehmigung)

dem Einfluss der Glaukomdiagnose auf die Effektivität beider Verfahren [16].

\section{Fazit für die Praxis}

- Auch wenn bisher keine umfangreichen Langzeitstudien oder vergleichenden Studien für die trabekuläre MIGS-Chirurgie mit Implantaten vorliegen, weisen die bisherigen Studienergebnisse und Real-worldDaten auf eine hinreichende IODSenkung hin.

- Indikationen stellen primäre und sekundäre OWG mit erhöhtem IOD dar, bei denen unter lokaler Therapie keine hinreichende Drucksenkung erreicht wird oder Nebenwirkungen bzw. eine unzureichende Adhärenz oder Persistenz eine Reduzierung der Medikamentenlast notwendig machen.

- Die trabekuläre Chirurgie mit Mikrostents weist ein sehr gutes Sicherheitsprofil auf. Abgesehen vom Mikrohyphäma sind bisher keine weiteren Komplikationen bekannt.

- Entscheidend für den Erfolg der MIGS-Chirurgie mit Implantaten sind die richtige Indikationsstellung und die postoperative Bewertung der Qualität der Implantation.

- Die Erfassung der MIGS-Eingriffe in gemeinsamen MIGS-Registern, die Identifizierung von Risikofaktoren für das operative Vorgehen und das postoperative Ergebnis stellen Herausforderungen für die kommende Zeit dar.

\section{Korrespondenzadresse

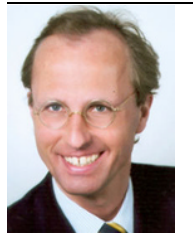 \\ Prof. Dr. A. G. M. Jünemann, F.E.B.O. \\ Klinik für Augenheilkunde, Universitätsmedizin Rostock, Universität Rostock Doberaner Str. 140, 18059 Rostock, Deutschland anselm.juenemann@med.uni- rostock.de}

\section{Einhaltung ethischer Richtlinien}

Interessenkonflikt. A.G.M. Jünemann ist als Referent für die Firma Glaukos Coperation, Inc. Laguna, Hills, CA, USA, tätig. R. Rejdak und B. Hohberger geben an, dass kein Interessenkonflikt besteht.

Dieser Beitrag beinhaltet keine von den Autoren durchgeführten Studien an Menschen oder Tieren.

Open Access Dieser Artikel wird unter der Creative Commons Namensnennung 4.0 International Lizenz (http://creativecommons.org/licenses/by/4.0/deed. de) veröffentlicht, welche die Nutzung, Vervielfältigung, Bearbeitung, Verbreitung und Wiedergabe in jeglichem Medium und Format erlaubt, sofern Sie den/die ursprünglichen Autor(en) und die Quelle ordnungsgemäßnennen, einen Linkzur Creative Commons Lizenz beifügen und angeben, ob Änderungen vorgenommen wurden.

\section{Literatur}

1. Ahmed I, Katz LJ, Chang DF et al (2014) Prospective evaluation of microinvasive glaucoma surgery with trabecular microbypass stents and prostaglandin in open-angle glaucoma. J Cataract Refract Surg 40:1295-1300

2. Arriola-Villalobos $P$, Martinez-De-La-Casa JM, Diaz-Valle D et al (2012) Combined iStent trabecular micro-bypass stent implantation and phacoemulsification for coexistent open-angle glaucoma and cataract: a long-term study. $\mathrm{Br} J$ Ophthalmol 96:645-649

3. Arriola-Villalobos P, Martinez-De-La-Casa JM, DiazValle D et al (2013) Mid-term evaluation of the new Glaukos iStent with phacoemulsification in coexistent open-angle glaucoma or ocular hypertension and cataract. Br J Ophthalmol 97:1250-1255

4. Belovay GW, Naqi A, Chan BJ et al (2012) Using multiple trabecular micro-bypass stents in cataract patients to treat open-angle glaucoma. J Cataract Refract Surg 38:1911-1917

5. Buchacra O, Duch S, Milla E et al (2011) One-year analysis of the iStent trabecular microbypass in secondary glaucoma. Clin Ophthalmol 5:321-326

6. Caprioli J, Kim JH, Friedman DS et al (2015) Special commentary: Supporting innovation for safe and effective minimally invasive glaucoma surgery summary of a joint meeting of the American Glaucoma Society and the Food and Drug Administration, Washington, DC, February 26,2014. Ophthalmology 122:1795-1801 
7. Craven ER (2015) Trabecular micro-bypass shunt (iStent $(R))$ : basic science, clinical, and future. Middle East Afr J Ophthalmol 22:30-37

8. Craven ER, Katz LJ, Wells JM et al (2012) Cataract surgery with trabecular micro-bypass stent implantation in patients with mild-to-moderate open-angle glaucoma and cataract: two-year follow-up. J Cataract Refract Surg 38:1339-1345

9. Donnenfeld ED, Solomon KD, Voskanyan L et al (2015) A prospective 3-year follow-up trial of implantation of two trabecular microbypass stents in open-angle glaucoma. Clin Ophthalmol 9:2057-2065

10. Fea AM(2010) Phacoemulsification versus phacoemulsification with micro-bypass stent implantation in primary open-angle glaucoma: randomized double-masked clinical trial. JCataract Refract Surg 36:407-412

11. Fea AM, Ahmed I, Lavia C et al (2017) Hydrus microstent compared to selective laser trabeculoplasty in primary open angle glaucoma: one year results. Clin Experiment Ophthalmol 45:120-127

12. Fea AM, Belda Jl, Rekas M et al (2014) Prospective unmasked randomized evaluation of the iStent inject $((\mathrm{R})$ ) versus two ocular hypotensive agents in patients with primary open-angle glaucoma. Clin Ophthalmol 8:875-882

13. Fea AM, Consolandi G, Zola M et al (2015) Microbypass implantation for primary open-angle glaucoma combined with phacoemulsification: 4-year follow-up. J Ophthalmol. https://doi.org/ $10.1155 / 2015 / 795357$

14. Ferguson TJ, Swan R, Ibach Metal (2017) Trabecular microbypass stent implantation with cataract extraction in pseudoexfoliation glaucoma. J Cataract Refract Surg 43:622-626

15. Fernandez-Barrientos Y, Garcia-Feijoo J, MartinezDe-La-Casa JM et al (2010) Fluorophotometric study of the effect of the glaukos trabecular microbypass stent on aqueous humor dynamics. Invest Ophthalmol Vis Sci 51:3327-3332

16. Gandolfi SA, Ungaro N, Ghirardini S et al (2016) Comparison of surgical outcomes between Canaloplasty and Schlemm's Canal Scaffold at 24 months' follow-up. J Ophthalmol. https://doi.org/ 10.1155/2016/3410469

17. Gonnermann J, Bertelmann E, Pahlitzsch M et al (2017) Contralateral eye comparison study in MICS \& MIGS: Trabectome(R) vs. iStent inject(R). Graefes Arch Clin Exp Ophthalmol 255:359-365

18. Hoeh H, Vold SD, Ahmed IK et al (2016) Initial clinical experience with the cypass micro-stent safety and surgical outcomes of a novel supraciliary microstent. J Glaucoma 25:106-112

19. Hohberger B, MonczakE, Mardin CY (2017)26years of the Erlangen glaucoma registry: demographic and perimetric characteristics of patients through the ages. Klin Monbl Augenheilkd. https://doi.org/ 10.1055/s-0043-112856

20. Hohberger B, Welge-Luen UC, Lammer R (2017) ICE-syndrome: a case report of implantation of a microbypass xen gel stent after DMEK transplantation. JGlaucoma 26:e103-e104

21. Katz LJ, Erb C, Carceller GA et al (2015) Prospective, randomized study of one, two, or three trabecular bypass stents in open-angle glaucoma subjects on topical hypotensive medication. Clin Ophthalmol 9:2313-2320

22. Klamann MK, Gonnermann J, Pahlitzsch M et al (2015) iStent inject in phakic open angle glaucoma. Graefes Arch Clin Exp Ophthalmol 253:941-947

23. Leske MC, Heijl A, Hussein M et al (2003) Factors for glaucoma progression and the effect of treatment: the earlymanifest glaucoma trial. ArchOphthalmol 121:48-56

24. Lewis RA (2014) Ab interno approach to the subconjunctival space using a collagen glaucoma stent. J Cataract Refract Surg 40:1301-1306

25. Lindstrom R, Lewis R, Hornbeak DM et al (2016) Outcomes following implantation of two second generation trabecular micro-bypass stents in patients with open-angle glaucoma on one medication: 18-month follow-up. Adv Ther 33:2082-2090

26. Neuhann TH (2015) Trabecular micro-bypass stent implantation during small-incision cataract surgery for open-angle glaucoma or ocular hypertension: long-term results. J Cataract Refract Surg 41:2664-2671

27. Patel I, De Klerk TA, Au L (2013) Manchester iStent study: early results from a prospective UK case series. Clin Experiment Ophthalmol 41:648-652

28. Pfeiffer N, Garcia-Feijoo J, Martinez-De-La-Casa JM et al (2015) A randomized trial of a Schlemm's Canal microstent with phacoemulsification for reducing Intraocular pressure in open-angle glaucoma. Ophthalmology 122:1283-1293

29. Saheb H, Ahmed I (2012) Micro-invasive glaucoma surgery: current perspectives and future directions. Curr Opin Ophthalmol 23:96-104

30. Samuelson TW, Katz LJ, Wells JM et al (2011) Randomized evaluation of the trabecular microbypass stent with phacoemulsification in patients with glaucoma and cataract. Ophthalmology 118:459-467

31. Spiegel D, Wetzel W, Neuhann T et al (2009) Coexistent primary open-angle glaucoma and cataract: interim analysis of a trabecular microbypass stent and concurrent cataract surgery. Eur J Ophthalmol 19:393-399

32. Sultan M, Blondeau P (2003) Episcleral venous pressure inyounger and oldersubjects in the sitting and supine positions. J Glaucoma 12:370-373

33. Tamm ER, Braunger BM, Fuchshofer R (2015) Intraocular pressure and the mechanisms involved in resistance of the aqueous humor flow in the trabecular meshwork outflow pathways. Prog Mol Biol TransI Sci 134:301-314

34. Tan SZ, Au L (2016) Manchester iStent study: 3-year results and cost analysis. Eye 30:1365-1370

35. Vandewalle E, Zeyen T, Stalmans I (2009) The iStent trabecular micro-bypass stent: a case series. Bull Soc Belge Ophtalmol 311:23-29

36. Vold SD, Voskanyan L, Tetz M et al (2016) Newly diagnosed primary open-angle glaucoma randomized to 2 trabecular bypass stents or prostaglandin: outcomes through 36 months. Ophthalmol Ther 5:161-172

37. Voskanyan L, Garcia-Feijoo J, Belda Jl et al (2014) Prospective, unmasked evaluation of the iStent $(R)$ inject system for open-angle glaucoma: synergy trial. Adv Ther 31:189-201

38. Wellik SR, Dale EA (2015) A review of the iStent((R)) trabecular micro-bypass stent: safety and efficacy. Clin Ophthalmol 9:677-684

39. Gierson I et al (2015) A novel Schlemm's Canal Scaffold: histologic observations. J Glaucoma 24:460-468

\section{Terminankündigung}

\section{8}

DOG 2018

Bonn, 27.09. - 30.09.2018

Ophthalmology 4.0

http://dog2018.dog-kongress.de/

\section{9}

AAD 2019

Düsseldorf, 12.03. - 16.03.2019

Kongressorganisation: Berufsverband der

Augenärzte e.V. (BVA)

http://www.aad.to/

DOG 2019

Berlin, 26.09. - 29.09.2019

http://www.dog.org/

2020

AAD 2020

Düsseldorf, 17.03. - 21.03.2020

Kongressorganisation: Berufsverband der

Augenärzte e.V. (BVA)

http://www.aad.to/

DOG 2020

Berlin, 01.10. - 04.10.2020

http://www.dog.org/ 